\title{
Le virus de la mosaïque du tabac chez le piment I. - Apparition en France du pathotype $\mathrm{P}^{1-2}$
}

\author{
Kahsay GEBRE SELASSIE, Robert DUMAS DE VAULX*, Georges MARCHOUX \& Edmond \\ POCHARD*
}

I.N.R.A., Station de Pathologie végétale, $\left(^{*}\right)$ Station d'Amélioration des Plantes, Centre de Recherches agronomiques d'Avignon, F 84140 Montfavet.

RÉSUMÉ
Virus,
Mosaique du Tabac,
Poivron,
Capsicum sp.,
Résistance.

\section{RESUME}

Capsicum sp.,

Résistance.

\begin{abstract}
Une nouvelle souche du virus de la mosaïque du tabac (VMT) a été isolée dans le Sud-Est de la France, à partir d'une variété de poivron commerciale «Lamuyo » portant le gène de résistance aux souches communes de VMT (pathotype $\mathbf{P}^{-0}$ ).

La nouvelle souche (pathotype $\mathrm{P}^{1-2}$ ) présente 4 caractéristiques biologiques originales :

- infection systémique des différents Capsicum résistants par hypersensibilité à la souche commune ;

- absence d'infection systémique de la tomate ;

- taille réduite des lésions locales chez différents hôtes hypersensibles aux 2 groupes de souches ;

- symptômes généralement atténués chez les hôtes sensibles aux 2 groupes de souches.

Toutes les origines de $C$. annuum et $C$. frutescens se sont révélées sensibles à la nouvelle souche, en revanche 4 lignées de C. chinense (PI 15225, PI 159230, PI 315008, PI 315023) sont apparues résistantes par hypersensibilité.

Les 2 dernières ont été retenues pour un croisement interspécifique avec certaines variétés de $C$. annuum. Les plantes $\mathrm{F} 1$ qui sont autostériles peuvent être croisées en retour avec l'un ou l'autre des parents et les produits sont autofertiles.
\end{abstract}

\section{SUMMARY}

Tobacco Mosaic Virus, Sweet pepper, Capsicum sp., Resistance.

\section{Apparition of $P^{1-2}$ strain of tobacco mosaic virus in peppers in France}

A new strain of Tobacco Mosaic Virus (TMV) was isolated from a commercial pepper variety "Lamuyo " which is normally resistant to common strain of TMV. Tomatoes, whether resistant or sensitive to common strain of TMV, were not infected with the new strain. The local lesions, with the new strain, were relatively very small, than those produced with our common strains. Similar strains, which have certain common biological properties have been already reported in Europe and U.S.A.

All tested varieties of $C$. annuum and $C$. frustescens were infected systemically; symptoms in $C$. frutescens were more severe than those of $C$. annuum.

Resistance by hypersensitivity was found in four C. chinense accessions (PI 15225, PI 159230, PI 315008 and PI 315023).

Crossing of $C$. annuum with $C$. chinense PI 315008 and PI 315023 was possible. $F_{1}$ plants were all resistant by hypersensitivity but sterile. However, back-crossing to either of the parents produced fertile progenies. Crossing was more easier with PI 315008 than with PI 315023.

\section{INTRODUCTION}

Le virus de la mosaique du tabac est l'une des plus graves maladies du poivron cultivé sous serre.

La résistance à ce virus est relativement commune à l'intérieur du genre Capsicum puisqu'elle a été trouvée chez C. annuum L., C. frutescens L., C. baccatum L. et $C$. chinense Jacq. (HolmES, 1937; COOK, 1959, 1963; POCHARD \& BREUILS, 1965 ; MO YENG LEE \& SMITH, 1968).

Cette résistance est liée à une réaction d'hypersensibilité déterminée par des gènes dominants ( $\mathrm{L} i$ chez $C$. annuum, $\mathrm{L}$ chez $C$. frutescens) appartenant à la même série allélique.
L'introduction de ces gènes de résistance dans certaines variétés commerciales avait ramené au second plan l'importance du VMT parmi les viroses affectant le poivron dans le Sud-Est de la France (MARChOuX et al., 1966).

Le danger est réapparu en 1978 avec la découverte, dans la région d'Avignon, d'une nouvelle souche de VMT capable d'attaquer les variétés et hybrides jusque-là résistants.

Le présent travail rapporte la mise en évidence chez cette souche de quelques propriétés biologiques originales et les premières données sur la recherche de nouveaux géniteurs de résistance.

Un second article portera sur le mode et l'importance de la transmission du virus par les semences et les méthodes de lutte à envisager. 


\section{MATÉRIEL ET MÉTHODES}

\section{A. Isolats et souches de VMT}

Les isolats de la nouvelle souche proviennent tous de la variété française «Lamuyo" résistante aux souches communes de VMT et cultivéc sous tunnels en plastique, d'une part à Villencuve-lès-Avignon (Gard) et d'autre part, à Châteaurenard (Bouches-du-Rhône).

Vingt prélèvements sont effectués à partir des jcunes feuilles de plants présentant des symptômes.

La souche commune de VMT utilisée comme référence est issue de piments contaminés naturellement dans la région d'Avignon. Cette souche qui appartient au groupe «tabac» porte le code Vi 76.

\section{B. Méthode d'inoculation et hôtes différentiels}

Pour chaque prélèvement, un poids frais de $1 \mathrm{~g}$ de tissus foliaires est broyé dans une solution $0,03 \mathrm{M}$ de $\mathrm{Na}_{2} \mathrm{HPO}_{4}$ additionnée de DIECA (2 p. 1000).

L'inoculation est effectuée par frottis au doigt protégé d'un doigtier en latex en présence de carborundum 400 mesh et de charbon végétal activé $(75 \mathrm{mg} / \mathrm{ml}$ de chacun des adjuvants).

Les plantes-tests, élevées et incubées en serres vitrées «insect-proof», sont inoculées à un stade juvénile : 23 feuilles. Les symptômes sont notés $4 \mathrm{j}$ après l'inoculation puis de façon hebdomadaire pendant 2 mois.

Nous avons utilisé la gamme d'hôtes réduite qui permet de distinguer par leurs réactions les 5 virus précédemment identifiés sur piment dans le Sud-Est de la France (MARCHOUX et al., 1965; MARCHOUX et al., 1976).

Dans une seconde étape et afin de préciser les propriétés biologiques des nouvelles souches de VMT, la gamme est étendue à d'autres Solanacées: Datura stramonium L., Lycopersicon esculentum Mill., Nicotiana clevelandii, $N$. glutinosa, $N$. tabacum, et à de nombreux cultivars de piment: C. annuum cv. «Doux d'Alger», «Sucette», «Turrialba », «Yolo Wonder», C. baccatum pendulum, $C$. chinense : 13 lignées dont «PI $315008 »($ ( Miscucho ») et "PI 315023 » ( Mishme »); C. frutescens : 10 lignées dont «Tabasco ».

\section{Recherche de lignées résistantes et croisements inter- spécifiques}

Quarante lignées entretenues à la Station d'Amélioration des plantes de Montfavet et appartenant à 4 espèces de Capsicum (C. annuum, $C$. baccatum, C. chinense et $C$. frutescens) ont été éprouvées vis-à-vis du VMT souche commune et souche nouvelle.

Des hybrides F1 ont été obtenus entre les 2 origines de $C$. chinense «Mishme " et "Miscucho» et 2 cultivars de C. annuum utilisés comme parents femelles:

— cv. «Doux des Landes» à petits fruits et sensible à la souche commune de VMT mais favorable à la réussite des hybrides interspécifiques (DUMAS DE VAULX \& PITRAT, 1977) ;

- hybride $F_{1}$ («Doux des Landes » $\times$ «Yolo Wonder »), résistant à la souche commune de VMT.

\section{RÉSULTATS}

\section{A. Symptômes naturels}

Les symptômes manifestés par le cultivar sensible "Lamuyo ", à la suite d'une contamination naturelle en serre par la nouvelle souche de virus, sont tardifs et se traduisent par une mosaïque en plages vert-jaune sur les feuilles du sommet de la plante. Les fruits de la première récolte présentent des défauts de coloration et un aspect bosselé (fig. 1). De plus, ces fruits ont la particularité de se détacher facilement. On observe également la coulure des boutons floraux qui réduit ou annule les récoltes ultérieures.

\section{B. Identification du virus}

Les propriétés symptomatologiques des isolats (notamment : lésions locales sur Datura stramonium, Nicotiana glutinosa et $N$. tabacum var. xanthi ; absence de réaction sur Vigna sinensis et Cucurbitacées) permettent d'identifier la nouvelle souche comme appartenant au VMT.

Cette identification est confirmée par l'observation au microscope électronique de particules en bâtonnets rigides de $15 \mathrm{~nm} \times 300 \mathrm{~nm}$.

\section{Caractérisation de la nouvelle souche de VMT}

La nouvelle souche de VMT se distingue de la souche commune par les caractéristiques biologiques suivantes:

1) Infection systémique des différentes variétés de Capsicum annuum dont "Yolo Wonder " et de toutes les lignées de C. frutescens, dont «Tabasco», toutes résistantes à la souche commune (tabl. 1);

2) Absence d'infection systémique chez la tomate qu'elle soit sensible (Monalbo) ou résistante (Momor) à la souche commune (tabl. 2) ;

3) Chez différents hôtes hypersensibles aux 2 types de souches, la taille des lésions locales induites par la nouvelle souche est plus petite $(<1 \mathrm{~mm})$ que celle des lésions produites par la souche commune $(1-5 \mathrm{~mm})$ chez les mêmes espèces: Datura stramonium, Nicotiana glutinosa et $N$. tabacum var. xanthi nc (tabl. 1).

4) Parmi 13 lignées de $C$. chinense, toutes résistantes à la souche commune, seulement 4 introductions: PI 315008 (Miscucho), PI 315023 (Mishme), PI 152225, PI 159236 sont résistantes par hypersensibilité à la nouvelle souche. A noter que chez cette espèce les lésions locales sont toujours de plus petite taille quelle que soit la souche (tabl. 2, fig. 2).

5) Chez les variétés de $C$. annuum, les symptômes provoqués par la nouvelle souche sont généralement peu accentués (type marbrure, tabl. 2).

Dans le même sens, la nouvelle souche n'induit qu'une infection latente des tabacs var. "Samsun » et " Paraguay ».

Par contre, sur $C$. frutescens les symptômes de mosaïque accompagnés de nécrose des tiges sont très accentués. (tabl. 2).

\section{Comportement des hybrides interspécifiques}

Les plantes $F_{1}$, obtenues à partir du croisement entre les 2 cultivars de $C$. annuum (utilisés comme parents femelles) et les 2 origines de $C$. chinense («Miscucho» et 




Figure 1

Symptômes sur fruit de la variété "Lamuyo " affectée par le VMTpathotype $P^{l \cdot 2}$.

Fruit symptoms on "Lamuyo" sweet pepper variety infected with TMV P $P^{1-2}$ strain.

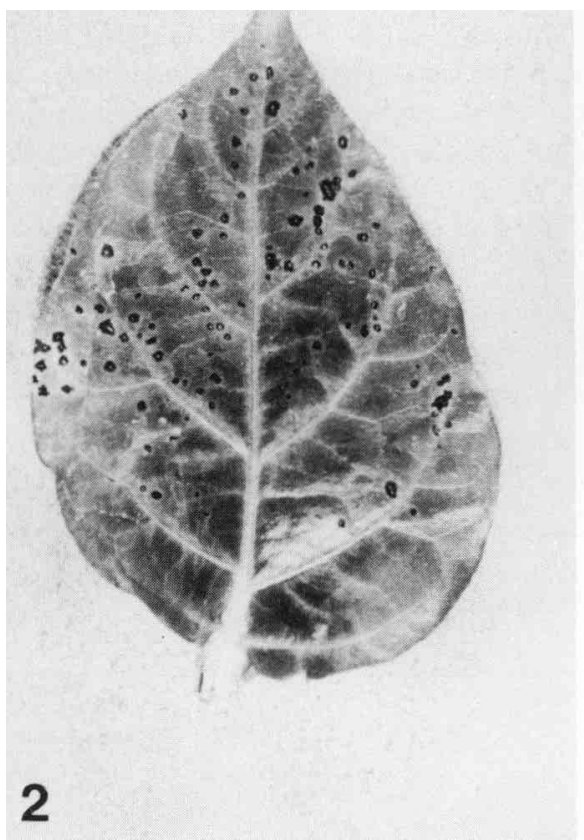

Figure 2

Lésions locales de petite taille produites par le VMT $P^{1 \cdot 2}$ inoculé sur une feuille de $\mathrm{C}$. chinense, PI 315008.

Local lesions of small size on C. chinense (PI 315008) infected with $T M V P^{l-2}$ strain.

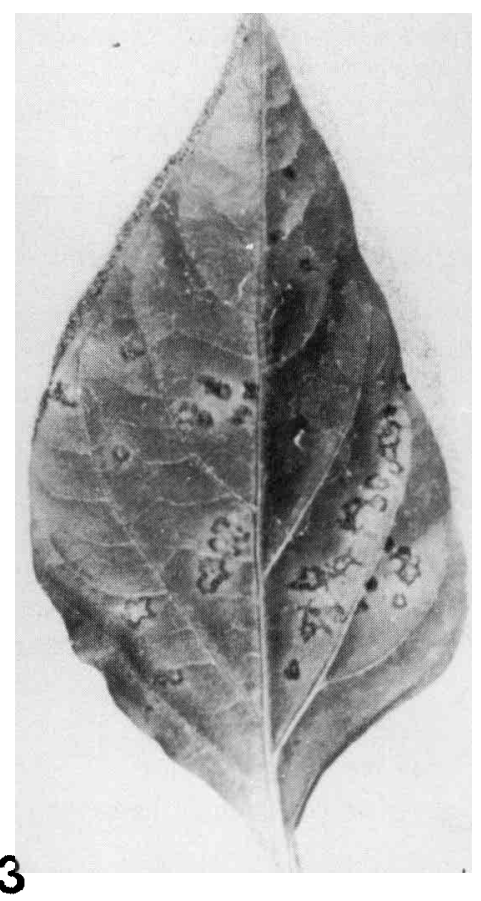

Figure 3

Lésions locales induites par VMT $P^{o}$ sur une feuille de l'hybride interspécifique C. annuum ("Doux des Landes" $\times$ "Yolo Wonder $») \times$ C. chinense (PI 315008).

Local lesions of medium size, on leaves of $F^{l}$ hybride C. annuum ("Doux des Landes" $\times$ "Yolo Wonder") $\times$ C. chinense $(P I$ 315008) with $T M V P^{0}$ (Vi-76 strain).

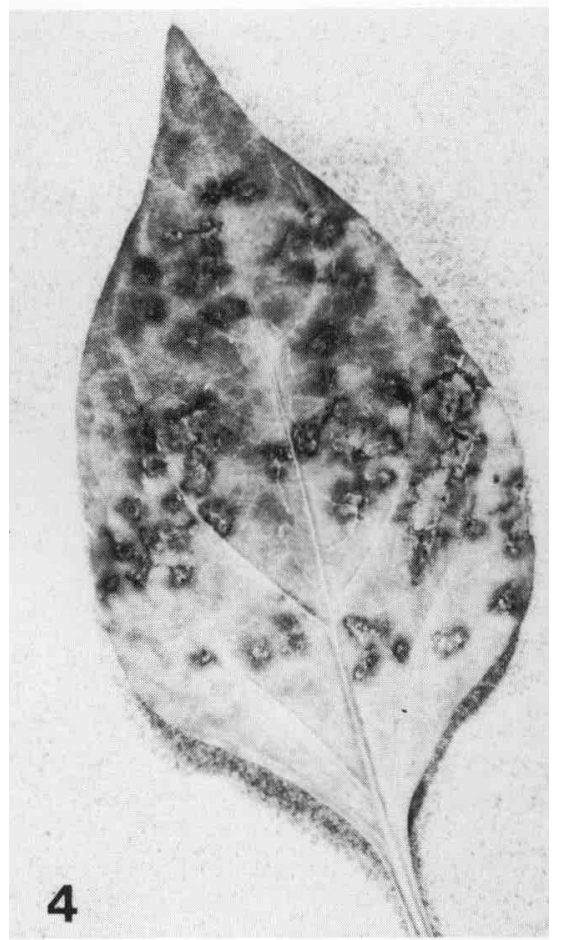

Figure 4

Lésions locales induites par VMT $P^{o}$ sur $\mathrm{C}$. annuum ( Doux des Landes " $x$ "Yolo Wonder").

Local lesions on C. annuum ("Doux des Landes" $\times$ "Yolo Wonder ") with $T M V-P^{\circ}(V \mathrm{i}-76$ strain). 


\section{TABLEAU 1}

Symptômes provoqués par la nouvelle souche de VMT sur les variétés de piment résistantes aux souches communes

Pepper varieties responses after inoculation by the new and common strains of $T M V$

\begin{tabular}{|c|c|c|}
\hline Espèces, Variétés ct Lignées & Souche nouvelle & $\begin{array}{l}\text { Souche commune } \\
\text { Vi } 76\end{array}$ \\
\hline $\begin{array}{l}\text { C. annuum Doux d'Alger } \\
\text { Sucette } \\
\text { Turrialba } \\
\text { Yolo Wonder } \\
\text { C. baccatum pendulum 3-4 } \\
\text { C. chinense } 9 \text { lignées } \\
\text { PI } 315008=\text { Miscucho } \\
\text { PI } 315023=\text { Mishme } \\
\text { PI } 152225 \\
\text { PI } 159236 \\
\text { C. frutescens } 10 \text { lignées } \\
\text { dont Tabasco }\end{array}$ & $\begin{array}{l}-/ \mathrm{m}\left({ }^{*}\right) \\
-/ \mathrm{m} \\
-/ \mathrm{m} \\
\mathrm{SpCN} / \mathrm{SkSpCN} \\
\frac{\mathrm{m}}{\mu \mathrm{ll} /-(-)} \\
\mu \mathrm{ll} /-(-) \\
\mu \mathrm{ll} /-(-) \\
\mathrm{ab} / \mathrm{Msk}\end{array}$ & $\begin{array}{c}\mathrm{LL} /- \\
\mathrm{LL} /- \\
\mathrm{LL} /- \\
\left.\mathrm{LL} /-)^{* *}\right) \\
\mathrm{LL} / \mathrm{L}- \\
\mu \mathrm{ll} /-(-) \\
\mu \mathrm{ll} /-(-) \\
\mu \mathrm{ll} /-(-) \\
\mu 1 \mathrm{l} /-(-) \\
\mathrm{ll} /-\end{array}$ \\
\hline
\end{tabular}

(*) Réaction locale/réaction systémique

$\left.{ }^{* *}\right)$ Résultat positif en condition de températures élevées.

$\mathrm{ab}=\mathrm{abscission}$

LL ou $\mathrm{ll}$ ou $\mu \mathrm{ll}=$ lésions locales grandes ou moyennes ou petites

$\mathrm{m}=$ marbrure

$\mathbf{M}=$ mosaïque

Sk = nécrose de la tige

$-=$ pas de réaction

(一) $(+)=$ rétro-inoculation négative ou positive

$\left({ }^{*}\right)$ Local reaction/systemic reaction

$\left({ }^{* *}\right)$ Positive result in high temperature conditions.

$\mathrm{ab}=$ abscission

$\mathrm{LL}$ or 11 or $\mu \| l l=$ large or normal or « pin-point " local lesion

$\mathrm{m}=$ mottle

$\mathbf{M}=$ mosaic

$\mathrm{Sk}=$ streak necrosis

$-=$ no reaction

$(-)(+)=$ bac inoculation : negative or positive

\section{TABLEAU 2}

Symptômes provoqués par la nouvelle souche et la souche commune de VMT sur quelques solanacées

Symptoms on some solanaceous plants to infection with new and common strain of $T M V$

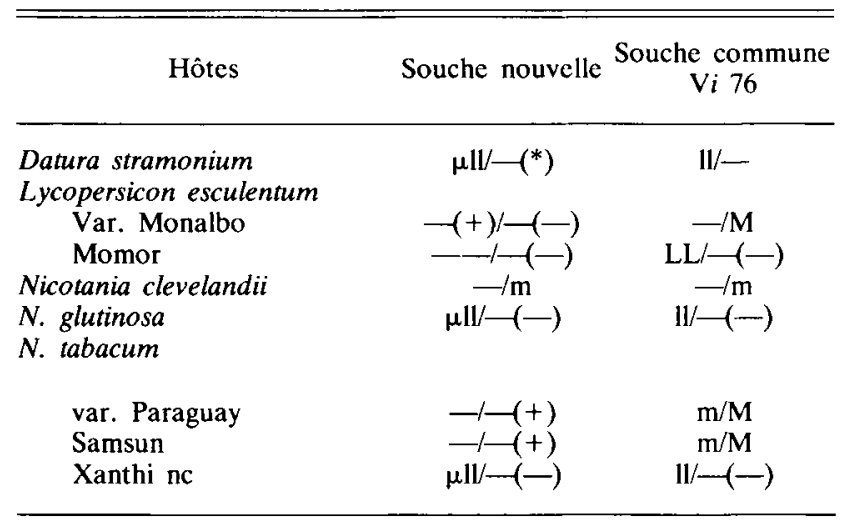

(*) Légende cf. tableau 1.

« Mishme») sont résistantes par hypersensibilité à la souche commune et les lésions locales sont de plus petite taille que celles qui apparaissent sur "Yolo Wonder" (fig. 3 et 4 ).
Les plantes $F_{1}$ issues du croisement $C$. annuum ( Yolo Wonder") $\times C$. chinense ("Miscucho" et "Mishme") sont résistantes par hypersensibilité à la nouvelle souche de VMT.

Les hybrides n'ont pas donné de descendance en autofécondation manuelle et seuls les hybrides avec "Miscucho" ont pu être rétrocroisés par $C$. annuum. Parmi les plantes de ce dernier rétrocroisement, nous en avons observées 34 sensibles et 22 résistantes à la nouvelle souche de VMT. La plupart des plantes $\mathrm{BC}_{1}$ ont été fertiles.

\section{DISCUSSION}

Un nouveau type de souches de VMT capables d'attaquer les variétés et hybrides de poivron jusque-là résistants est apparu ces dernières années dans le Sud-Est de la France. Un phénomène comparable a été noté dans plusieurs pays, en Hollande (RAST, 1977) et en Roumanie (POP, 1979).

On peut penser que l'utilisation de variétés à résistance monogénique a conduit à l'apparition puis à l'extension progressive des pathotypes adaptés à ce gène.

Si on désigne par les symboles $\mathrm{L}^{1}$ (anciennement $\mathrm{L}^{\mathrm{i}}$ ) et $\mathrm{L}^{2}$ (anciennement $\mathrm{L}$ ) les allèles de résistance portés respectivement par «Yolo Wonder» (C. annuum) et « Tabasco » $(C$. frutescens), les nouvelles souches isolées en Provence appartiendraient au pathotype $\mathrm{P}^{1-2}$ (BoukEMA et al., 1980) (tabl. 3).

Les variétés sensibles à la souche commune présentent généralement des symptômes moins accusés avec la nouvelle souche. Ainsi, chez la nouvelle souche (pathotype $\mathrm{P}^{\mathrm{l}-2}$ ), le gain en virulence parait s'accompagner d'une certaine perte d'agressivité à l'égard du piment. Cette remarque s'étend également à la tomate qui échappe à l'infection par le pathotype $\mathrm{P}^{1-2}$ et aux tabacs var. «Samsun" et "Paraguay " dont l'infection est latente.

L'existence de telles souches dites "Samsun latent " ou SLTMV a été montrée depuis de nombreuses années aux Etats-Unis (MC KINNEY, 1952; GREENLEAF et al., 1964).

Une autre caractéristique remarquable de cette nouvelle souche est la taille réduite des lésions locales qu'elle provoque chez la plupart des Solanées hypersensibles. Cette propriété a également été notée chez les souches "SLTMV " et certaines souches atypiques isolées de tomate

\section{TABLEAU 3}

Relations gène pour gène chez le couple virus de la mosaïque du tabac-piment (d'après BouktMA et al., 1980)

Strains of TMV and genes for resistance (after BOUKEMA ct al., 1980)

\begin{tabular}{|c|c|c|c|c|}
\hline \multirow{2}{*}{ Pathotypes du TMV } & \multicolumn{4}{|c|}{ Allèles de résistance } \\
\hline & $\mathbf{L}^{+} \mathbf{L}^{+}$ & $\mathbf{L}^{1} \mathbf{L}^{1}$ & $\mathrm{~L}^{2} \mathrm{~L}^{2}$ & $\mathrm{~L}^{3} \mathrm{~L}^{3}$ \\
\hline$P^{0}$ & $S\left({ }^{*}\right)$ & $\mathrm{H}$ & $\mathrm{H}$ & $\mathbf{H}$ \\
\hline$P^{1}$ & $\mathbf{S}$ & $\mathrm{S}$ & $\mathrm{H}$ & $\mathrm{H}$ \\
\hline $\mathrm{P}^{1-2}$ & $S$ & $\mathrm{~S}$ & $\mathrm{~S}$ & $\mathbf{H}$ \\
\hline $\mathrm{P}^{1-2-3}$ & $\mathrm{~S}$ & $S$ & $S$ & $\mathrm{~S}$ \\
\hline
\end{tabular}

(*) $\mathrm{S}=$ sensibilité : mosaïque

$\mathrm{H}=$ résistance par hypersensibilité : lésions locales.

(*) $\mathrm{S}=$ sensibility : mosaic

$\mathrm{H}=$ resistance by hypersensibility: local lesion. 
(Miller \& THORNBERRY, 1958) et retrouvée chez un mutant obtenu après traitement à l'acide nitreux (DAHL \& KNIGHT, 1963).

\section{CONCLUSION}

L'apparition d'un nouveau pathotype, VMT-P ${ }^{1-2}$, dans les cultures sous abri du Sud-Est de la France nécessite de compléter l'étude des solutions génétiques, satisfaisantes jusque-là.

Du matériel résistant issu du croisement interspécifique
$C$. annuum $\times C$. chinense a été obtenu et peut être exploité pour la sélection du piment.

Néanmoins, l'apparition possible de souches encore plus virulentes attaquant les sources de résistance provenant de C. chinense : pathotype $\mathrm{P}^{1-2 \cdot 3}$ (tabl. 3 : STOENESCU, 1978 ; BoukEMA et al., 1980) nécessite, d'une part la recherche d'autres sources de résistance et, d'autre part, l'observation de mesures prophylactiques.

\section{REMERCIEMENTS}

Nous remercions MM. LOT et MARROU pour leurs conseils au cours de ce travail et M. ROUGIER pour les photographies.

\section{RÉFÉRENCES BIBLIOGRAPHIQUES}

Boukema I. W., 1977. Resistance in Capsicum to a pepper strain of TMV. Proc. Third Capsicum Eucarpia Mceting. Avignon-Montfavet $85-88$

Boukema I. W., Jansen K., Hofman K., 1980. Strains of TMV and genes for resistance in Capsicum. Proc. Fourth Capsicum Eucarpia Meeting, Wageningen 44-48.

Cook A. A., 1959. Multiple virus disease resistance in a strain of Capsicum annuum. Phytopathology, 49, 198-201.

Cook A. A, 1963. Dominant resistance to tobacco mosaic virus in an exotic pepper. Plant Dis. Rep., 47, 783-786.

Dahl Doris, Knight C. A., 1963. Some Nitrous Acid-Induced Mutants of Tomato Atypica Mosaic Virus. Virology 21, 580-586.

Dumas de Vaulx R., Pitrat M., 1977. Interspecific Hybridization in Plant Breeding. Proc. $8^{\text {th }}$ Congress Eucarpia. Madrid 327-333.

Feldman J. M., Oremianer S., 1972. An unusual strain of tobacco mosaic virus from pepper. Phytopathol. Z., 75, (3), 250-267.

Greenleaf W. H., Cook A. A., Heyn A. N. J., 1964. Resistance to tobacco mosaic virus in Capsicum with reference to the Samsun latent strain. Phytopathology, 54, 1367-1371.

Holmes F. O., 1937. Inheritance of resistance to tobacco mosaic disease in the pepper. Phytopathology, 27, 637-642.

Marchoux G., Gebre S. K., Quiot J. B., 1976. Observations préliminaires concernant les souches et les plantes réservoirs du virus Y de la pomme de terre dans le Sud-Est de la France. Agric. Conspect. Scient., 39, (49), 541-552.

Marchoux G., Marrou J., Migliori A., 1965. Réaction du Poivron (Capsicum annuum L.) à quelques virus répandus dans les cultures maraîchères françaises méridionales. Ann. Epiphyt., 16, HS, $109-$ 117.

Marchoux G., Marrou J., Messiaen C. M., Pochard E., 1966. Les maladies à virus du piment et de l'aubergine dans les cultures françaises méridionales. Proc. Ist Congré U.P.M., BARI, 434-441.

Mc Kinney H. H., 1952. Two strains of tobacco mosaic virus, one of which is seed-borne in an etch-immune pungent pepper. Plant Dis. Rep., 36, 184-187.

Miller P. M., Thornberry H. H., 1958. A new viral disease of tomato and pepper. Phytopathology, 48, 665-670.

Mo-Yeong Lee, Smith P. G., 1968. Identification of the L gene for tobacco mosaic resistance in three pepper species. Phytopathology, 58, 1445.

Pochard E., Breuils G., 1965. La résistance du Piment (Capsicum annuum L.) à la mosaique du tabac et au virus 1 du concombre. Modalités et transmission héréditaire. $1^{\text {ères }} J$. Phytiatr. Phytopharm. Circum Méditer., Marseille 189-193.

Pop I. V., 1979. Cercetari privind atacul tulpinilor virusului mozaicului tutunului la ardeiul gras cultivat in sere. An. Inst. Cercet. Prot. Plant., Bucaresti, XV, 17-20.

Rast A. T. B., 1977. Introductory remarks on strains of TMV infecting peppers in the Netherlands. Proceeding Third Capsicum - Eucarpia Meeting, Avignon-Montfavet 83-84.

Stoenescu A. T., 1978. O nouà tulpinà a virusului mozaicului semnalatà în culturile de ardei din sere. Extras Prod. veg.-Hortic., 10, 13-17. 